
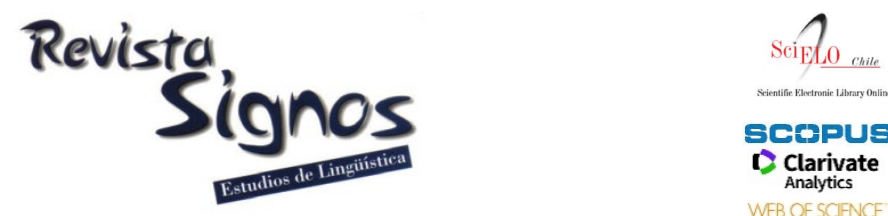

\title{
Análisis ideacional e interpersonal de escritos estudiantiles de historia en español dentro del marco sistémico
}

\section{Ideational and interpersonal analysis of student history texts in Spanish within the systemic framework}

\author{
Natalia Ignatieva \\ UNIVERSIDAD NACIONAL AUTÓNOMA DE MÉXICO \\ ESCUELA NACIONAL DE LENGUAS, LINGÜístiCA Y TRADUCCIÓN \\ MÉXICO \\ ignatiev@unam.mx
}

Recibido: 30-IX-2019 / Aceptado: 28-VIII-2020

DOI: $10.4067 /$ S0718-09342021000100169

\section{Resumen}

El presente trabajo busca explorar cómo ciertos tipos de procesos se relacionan con la expresión de la valoración en los textos académicos, nuestro objetivo es analizar la transitividad verbal y los aspectos evaluativos en la redacción académica de estudiantes de licenciatura en historia. La base teórica del estudio es la Lingüística SistémicoFuncional y, en particular, el Sistema de la Valoración. Presentamos aquí el análisis realizado a partir de uno de sus subsistemas: el de actitud. La investigación se basa en escritos estudiantiles que pertenecen a dos géneros (preguntas-respuestas y ensayos) en una misma disciplina (historia). Dentro del análisis ideacional exploramos tres tipos de procesos: verbales, relacionales y mentales, mientras que en el marco del análisis interpersonal clasificamos las cláusulas que expresan la valoración de acuerdo con los tres dominios de la actitud: afecto, juicio y apreciación. Los resultados preliminares muestran algunas similitudes entre los dos géneros como, por ejemplo, la prevalencia de procesos relacionales y la dominancia de la apreciación como el recurso preferido para expresar la actitud. Sin embargo, se encontraron también diferencias: por ejemplo, en los ensayos aumenta significativamente el porcentaje de cláusulas con evaluación negativa, al igual que el porcentaje de cláusulas que expresan juicio. Estos y otros hallazgos se presentarán dentro de una pequeña discusión sobre la interacción de los significados ideacionales e interpersonales y, consecuentemente, sobre los aspectos léxico-gramaticales y semántico-discursivos de textos académicos.

Palabras Clave: Transitividad, Valoración, Actitud, proceso, cláusula. 


\begin{abstract}
This work intends to explore how certain process types in Spanish are related to the expression of appraisal in academic texts, our objective is to analyze verbal transitivity and evaluative aspects of student academic writing on history. Our study draws on Systemic Functional Linguistics and, in particular, on the Appraisal System, which explores evaluative meanings in language, here we shall analyze only one of its subsystems, attitude. The analysis is based on student texts belonging to two genres (question-answer and essay), but to only one discipline (history). Within the ideational analysis we explored three types of processes in academic texts: verbal, mental and relational while within the interpersonal framework we classified the clauses that express appraisal according to the three domains of the attitude system: affect, judgment and appreciation. The preliminary results show certain similarities between the two genres, for example, the prevalence of relational processes and the dominance of appreciation as the preferred means of expressing attitude. However, some differences were also found, for example, in the essays the percentage of clauses with negative evaluation grows considerably, and so does the number of clauses expressing judgment. These and other findings will be presented within a small discussion about the interaction of ideational and interpersonal meanings and, consequently, about the lexicogrammatical and semantic-discursive aspects of academic texts.
\end{abstract}

Key Words: Transitivity, Appraisal, Attitude, process, clause.

\title{
INTRODUCCIÓN
}

Este trabajo se enmarca dentro del proyecto Tipología verbal y evaluación en la escritura académica de las humanidades ${ }^{1}$ que se lleva a cabo en la Universidad Nacional Autónoma de México (UNAM) y que, a su vez, forma parte del proyecto internacional Systemics Across Languages ( $S A L$ ) sobre procesos verbales. Nuestro equipo de investigación había participado antes en el $S A L$ Project con un análisis léxico-gramatical de cláusulas verbales en el lenguaje académico en español (Ignatieva, 2011; Ignatieva \& Zamudio, 2012; Ignatieva \& Rodríguez-Vergara, 2015). El presente proyecto extiende su análisis a otros tipos de procesos y busca explorar cómo éstos se relacionan con la expresión de la valoración en las cláusulas que los contienen. Nuestra intención es analizar estos procesos y su conexión con los aspectos valorativos de la escritura a través de diferentes géneros (pregunta-respuesta, ensayo, reseña, etc.) y disciplinas (literatura, historia, geografía). Estamos usando textos estudiantiles del Corpus CLAE (Corpus del Lenguaje Académico en Español), producto de nuestros estudios anteriores (Ignatieva \& Colombi, 2014).

Martin y White (2005) en su análisis de diferentes tipos del discurso mencionan la relación entre los significados ideacionales e interpersonales, misma que buscamos en este trabajo. De acuerdo con esto, nuestro proyecto tiene que ver con dos niveles de análisis: léxico-gramatical y semántico-discursivo, y con dos sistemas: transitividad y valoración. Aunque hay estudios que intentan conectar algunos tipos de procesos con diferentes formas de evaluación (Hyland, 1999; Chen, 2007; Lavid, 2008, etc.), estos 
no tratan el problema desde el ángulo adoptado en nuestro trabajo. Además, la mayoría de los estudios toman el inglés como base de su análisis.

En este artículo, el objetivo es analizar la transitividad verbal y los aspectos evaluativos en tres tipos de procesos: verbales, mentales y relacionales; escogimos los mentales y relacionales por su proximidad a los procesos verbales en la famosa rueda de la portada de la segunda edición de Introduction to Functional Grammar (Halliday, 1994) (ver Figura 1). Vamos a explorar textos estudiantiles pertenecientes a dos géneros académicos (pregunta-respuesta y ensayo) en una misma disciplina (historia). El punto de partida será el análisis de transitividad seguido por el análisis de valoración en la búsqueda de la interacción entre ambos. Nuestra meta es observar cómo se desenvuelvan significados interpersonales en la redacción académica de estudiantes de historia y como los escritores utilizan diferentes opciones para codificar las categorías semánticas de actitud.

\section{Bases teóricas}

Tanto el proyecto en que se inserta este estudio como el análisis realizado aquí se basan en la Lingüística Sistémico Funcional, propuesta originalmente por Halliday $(1978,1994)$ y desarrollada posteriormente por otros investigadores dentro del área (Martin, 1992; Martin \& Rose, 2003; Matthiessen, 1995; Thompson, 1996; Ghio \& Fernández, 2008; Oteíza Silva, 2006; Montemayor-Borsinger, 2009; Lavid, Arús \& Zamorano, 2010, entre otros). Dentro de este marco se presenta un modelo holístico del lenguaje y su contexto social que concibe el lenguaje como un recurso de construcción de significados. El lenguaje hace posible nuestra interpretación e interacción con el mundo natural y el social y, consecuentemente, el lenguaje resulta ser un aspecto necesario de la vida humana en todas sus modalidades. Esta teoría considera el lenguaje como una red de sistemas, formados a su vez por subsistemas de carácter funcional, derivados del uso que se da al lenguaje dentro de un contexto social.

Para proporcionar una visión global de fenómenos lingüísticos el modelo sistémico funcional describe el lenguaje desde múltiples perspectivas, una de las más importantes sería la funcional. Halliday (1994) considera el lenguaje como un sistema de significados condicionados por sus funciones, las llama 'metafunciones', i. e. las funciones más generales y abstractas que son comunes para todas las lenguas humanas. Son tres: la 'metafunción ideacional' que refleja la realidad del mundo y nuestras experiencias en él, la 'interpersonal' que tiene que ver con los participantes de la interacción, sus opiniones, emociones, etc. y la 'textual' que organiza el texto. Estas metafunciones nos permiten expresar tres tipos de significados: ideacionales, interpersonales y textuales (Halliday \& Matthiessen, 1999). 


\subsection{El sistema de transitividad}

Los significados ideacionales y, en particular, los experienciales, son aquellos que el hablante o escritor manipula para codificar sus experiencias en el mundo exterior e interior. Se materializan en la cláusula por medio de un sistema lingüístico de transitividad que construye el mundo de experiencia a través de un inventario de tipos de procesos. En términos del potencial de significado, "la transitividad es el componente ideacional en las cláusulas" (Halliday, 1976: 21). Este sistema construye gramaticalmente la experiencia con toda su complejidad, en el mundo afuera y dentro de nosotros, a partir de diversos tipos de procesos que se pueden realizar (García, 2013). Alrededor de cada tipo de proceso se organizan diferentes combinaciones de participantes en cada cláusula. Estas combinaciones de participantes y procesos son las que permiten representar todo tipo de experiencias y con ello ordenar la diversidad de eventos que ocurren de manera constante (the flow of events) (Halliday \& Matthiessen, 2004).

El Sistema de transitividad es, entonces, el que permite construir y organizar las diversas experiencias. Es necesario señalar que, originalmente, Halliday (1968) establece tres tipos de procesos: los 'materiales', los 'mentales' y los 'relacionales'. Posteriormente, a éstos le suma tres tipos más: los procesos 'verbales', los procesos 'conductuales' y los 'existenciales' (Halliday, 1994). Los tres primeros son considerados procesos básicos mientras que los tres posteriores son vistos como procesos intermedios.

Los procesos verbales, como procesos intermedios, se sitúan entre los procesos mentales y los relacionales puesto que una relación simbólica, que se construye en la conciencia humana, se manifiesta en el lenguaje como el decir (Halliday, 2004). Cabe señalar, sin embargo, que Matthiessen (1995) considera que son cuatro los tipos básicos de proceso. A los tres tipos propuestos por Halliday (materiales, mentales y relacionales) añade los verbales como otro proceso básico ya que, señala, éstos tienen características propias que los diferencian y separan de otros tipos de proceso. En el presente estudio, hemos seguido el punto de vista propuesto por Matthiessen, ya que los datos de nuestros proyectos anteriores confirman su posición teórica (Para más detalles, ver Ignatieva, 2016). Los seis tipos de procesos se puede apreciar en la Figura 1. 


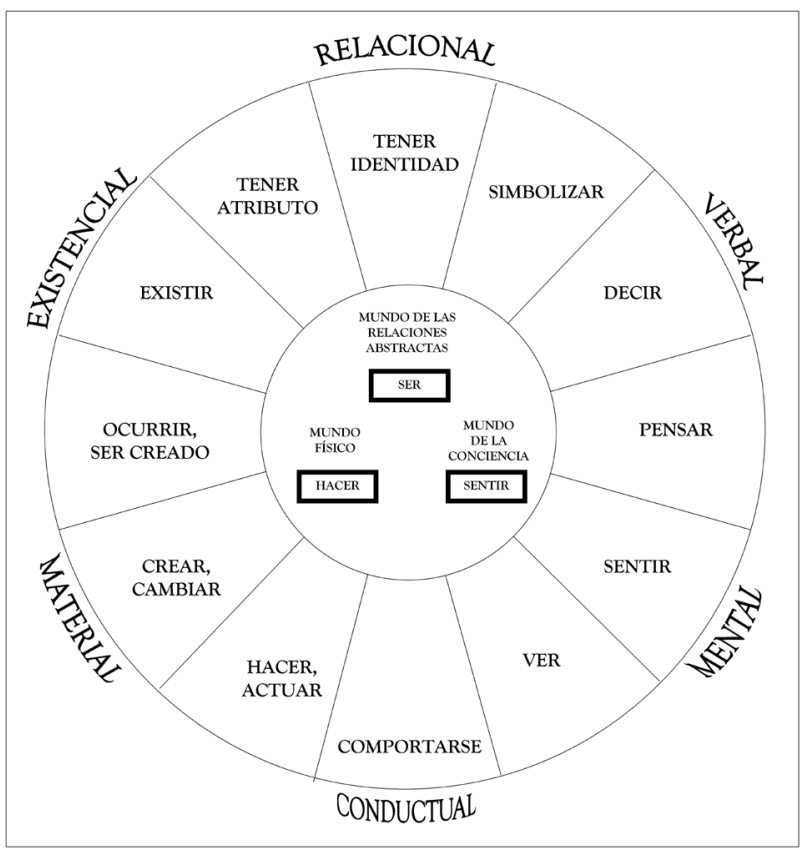

Figura 1. Tipos de procesos verbales según Halliday (1994).

Como mencionamos, cada tipo de proceso conlleva diferentes participantes formando así su propio modelo -o esquema- con el que se construye la cláusula (Lavid et al., 2010). Vale la pena subrayar, además, que en la LSF la transitividad no es una categoría del verbo (como en la gramática tradicional) sino un sistema de cláusula realizado a nivel léxico-gramatical (Matthiessen, 1999).

\subsection{El Sistema de Valoración (Appraisal)}

Los significados interpersonales tienen que ver con las relaciones sociales, es decir, cómo las personas interactúan, cómo expresan e intercambian sus opiniones. Sus sistemas básicos son modalidad que define el tipo de intercambio (dar y pedir información o dar y pedir servicios) y valoración (Martin \& White, 2005; Hood, 2010). De esta manera, la aproximación metafuncional hacia el análisis de datos es importante dado que este enfoque puede facilitarnos el reconocimiento de las maneras de cómo 'significados interpersonales se relacionan con selecciones ideacionales en el discurso’ (Hood, 2010).

El Sistema de Valoración se puede considerar como una extensión en el estudio de los significados interpersonales realizados por Halliday (1994). Desarrollada dentro de la Escuela de Sydney (Martin, 1992; Martin \& White, 2005), el Modelo de la Valoración explora y describe de manera más detallada la expresión de actitud y posicionamiento a través de subcategorías establecidas y permite describir la intersubjetividad del hablante desde el estrato semántico-discursivo en términos de sistemas. 
Así, el Sistema de Valoración está dividido en tres subsistemas: actitud, compromiso y gradación (attitude, engagement and gradation). El subsistema de actitud refiere el posicionamiento del hablante con respecto a emociones, ética y estética. Como constructo, la actitud modela recursos lingüísticos que se agrupan bajo el término 'evaluación' en algunas otras teorías (e.g. Thompson \& Hunston, 2000). En cuanto al compromiso, este expresa cómo la voz textual se posiciona con respecto a otras voces, mientras que la gradación ayuda al hablante ajustar la intensidad de la valoración dentro de una escala. En este estudio analizaremos solo un subsistema, actitud.

El subsistema de actitud incluye opciones lingüísticas para expresar emociones (affect), para apreciar cosas y entidades abstractas usando criterios estéticos (appreciation) y para evaluar personas con base en criterios éticos (judgement) (Martin \& White, 2005). De acuerdo con esto, el subsistema de actitud también se divide en tres áreas: afecto, apreciación y juicio. Por otra parte, la actitud se caracteriza por la polaridad, la cual indica "el sistema positivo/negativo" (Matthiessen, Teruya \& Lam, 2010: 161), ilustraremos esto con los siguientes ejemplos:

(1) ... al historiador le debe interesar el hecho histórico, (P-R7)

(2) Por ultimo al igual que los autores anteriores muestra preocupación por el mal uso que la política hace de la historia, (En3)

(3) ... lo importante no es la duración del acontecimiento, sino la trascendencia ... (P-R1)

(4) El historiador debe tener presente que no todas las huellas del pasado son verdaderas, (P-R8)

(5) El historiador debe ser objetivo y honesto ante ambos aspectos, (En2)

(6) Sin embargo, sobre las operaciones historiográficas, Bloch las describe (según yo) muy ligeramente. (P-R10)

En los ejemplos (1) y (2) podemos observar las instancias de afecto, en el primero de estos, con la polaridad positiva mientras que en el segundo, con la polaridad negativa. Las oraciones (3) y (4) codifican la apreciación, igualmente la (3) muestra un valor positivo, y la (4), negativo. Finalmente, los ejemplos (5) y (6) presentan los casos de juicio, también con la polaridad distinta: positiva en (5) y negativa en (6).

En suma, el Modelo de Valoración provee 'una base para un análisis teóricamente informado de significados interpersonales' que se construye en los textos estudiantiles (Hood, 2010). Vale la pena anotar que la mayoría de investigaciones sobre la valoración se ha realizado analizando el inglés mientras que en español se han hecho relativamente pocos estudios todavía (pero se puede mencionar Moss \& Mizuno, 2011; Castro Azuara, 2013; Navarro, 2014; Zamudio, 2016; Rodríguez-Vergara \& Contijoch Escontria, 2016; Perales-Escudero, 2018, etc.). Sin embargo, es nuestra creencia que el Modelo de la Valoración en sus términos básicos tiene un potencial universal, i. e., se podría aplicarla al español o cualquier otra lengua. Entonces, existe la 
necesidad para más aplicaciones de este modelo con el fin de comprobar su total o parcial aplicabilidad y detectar alguna especificidad (si la hay) para lenguas particulares.

Por otra parte, nuestro interés en este trabajo se focaliza en la interacción de los sistemas de transitividad y de valoración y, consecuentemente, de los significados ideacionales e interpersonales.

\section{La realización de actitud}

La evaluación se puede expresar en el texto desplegando un amplio rango de recursos lingüísticos. De acuerdo con Thompson y Hunston (2000), cada tipo de evaluación requiere de sus propios recursos gramaticales $y$, por consiguiente, hay diferentes formas de codificar la valoración en la cláusula. Así, los procesos pueden realizar la evaluación intrínsicamente, por ejemplo, un significado actitudinal puede ser incorporado en el proceso mismo, e. g.:

(1) El texto que más me gustó fue el de Gurevich (En8),

donde actitud está expresada por el proceso 'gustar', y su significado interpersonal implica afecto con la polaridad positiva. Los procesos, en los cuales su significado ideacional se fusiona con el significado actitudinal, generalmente pertenecen al grupo de procesos mentales, Hunston (1995) los llama 'verbos de atribución', otro ejemplo sería (2):

(2) Lo que le interesa es señalar los hechos concretos (P-R9)

En (2) el proceso mental 'interesar' expresa afecto con la polaridad positiva, denotando algún tipo de inclinación. Otros grupos de procesos también pueden codificar la evaluación, e. g. algunos procesos verbales que toman la Meta como segundo participante en el acto de comunicación (cuando el primero es el Emisor), e. g.:

(3) ... y no les importa desinformarlos (a la gente) (En7)

En el ejemplo (3) tenemos el caso de juicio con la polaridad negativa expresada por el proceso 'desinformar (a alguien)'. Los procesos verbales como 'desinformar' representan una lista cerrada; Matthiessen (1995: 285) los llama "procesos de impacto verbal', este grupo incluye verbos como 'acusar', 'felicitar', 'elogiar', 'criticar', etc., los cuales no verbalizan el mensaje como Informe o locución (Caffarel, 2006), i. e. ellos lo incorporan en el significado del verbo.

Otra manera de realizar la evaluación es por medio de un grupo nominal (GN), e.g.:

(4) Pues de esta manera la certeza en las investigaciones es mayor (En9). 
En (4) el grupo nominal 'la certeza' se analiza como un ejemplo de apreciación con un valor positivo, en una cláusula relacional con el proceso 'ser'. Tanto sustantivos (en 4) como adjetivos (en 5) pueden codificar la actitud dentro de un grupo nominal, en el útimo caso el adjetivo funciona como epíteto ('honesta' y 'confiable') que modifica un sustantivo ('disciplina'). (5) también es un ejemplo de apreciación con una polaridad positiva, introducida por un proceso relacional ('ser'):

(5) Se busca por todos los medios que sea una disciplina honesta y confiable (En8).

Otra forma de incluir evaluación en la cláusula sería por medio de un atributo, e.g.:

(6) Para Bloch, la observación es importante. (P-R1)

(7) El uso público de la historia no es una práctica que se debe rechazar o considerar demoníaca por principio (En7)

En (6) se puede observar un proceso relacional ('ser') conectado con el atributo 'importante' que se analiza como un caso de apreciacion con una polaridad positiva mientras que en (7) un proceso mental 'considerar' junto con el atributo 'demoníaca' codifica afecto, pero con un valor negativo.

La evaluación puede también realizarse por medio de una cláusula, en la mayoría de los casos es una cláusula proyectada con un proceso verbal (8) o mental (9) en la cláusula proyectante, e. g.:

(8) Puedo asegurar que la historia... es una verdadera ciencia porque es explicativa... (En8).

(9) Entonces debe entenderse que la realidad histórica... es digna de ser tomada como objeto de estudio (P-R4)

Tanto el ejemplo (8) como el (9) presentan casos de apreciación con la polaridad positiva expresada en las cláusulas proyectadas por el proceso verbal 'asegurar' (en 8) y el proceso mental 'entenderse' (en 9).

Finalmente, la evaluación puede ser realizada con la ayuda de un adjunto, e.g.:

(10) La mayoría de las personas... consideran la labor del historiador como una inutilidad (En7).

(11) Sin embargo pensar en términos tan parcos nos traería problemas ( $\operatorname{En} 9)$.

En (10) el juicio con una polaridad negativa se asocia con el proceso mental 'considerar', mientras que en el (11) el proceso mental 'pensar' está acompañado por el adjunto 'en términos tan parcos' para expresar la apreciación con un valor negativo.

En suma, la realización lingüística de evaluación puede ser expresada por medio de: un proceso, un GN, un atributo, una cláusula y un adjunto. Vale la pena mencionar que escogimos este inventario de categorías lingüísticas, entre otros tantos posibles, aplicando los criterios que mejor reflejan los propósitos de nuestro estudio. Por 
ejemplo, 'la cláusula' como tal categoría solo tiene sentido si tomamos en cuenta la relación entre un verbo proyectante (verbal o mental) y la locución proyectada por este verbo. Por otra parte, tratamos de reducir el número de categorías en el inventario y así, por ejemplo, ‘el epíteto' se incluyó en la categoría del 'grupo nominal', esto nos permitió reducir nuestro inventario a cinco categorías.

\section{Metodología}

Como ya fue mencionado, usamos el corpus CLAE, i. e., textos estudiantiles recolectados en el Colegio de Historia de la Facultad de Filosofía y Letras de la Universidad Nacional Autónoma de México. Los textos pertenecen a una sola disciplina: historia, pero a dos géneros ${ }^{2}$ : ensayo (10 textos) y pregunta-respuesta (10 textos), que es una tarea que estudiantes hacen en el salón de clase como parte de su examen semestral. En su forma inicial los textos de pregunta-respuesta fueron escritos a mano y fueron digitalizados posteriormente por los investigadores, mientras que los textos del género ‘ensayo' fueron escritos en casa y entregados en la forma impresa. Vale la pena notar que los estudiantes no recibieron ninguna indicación genérica previa a la elaboración de estos textos.

El método usado en este estudio incluye tanto un análisis cuantitativo, como uno cualitativo. Cuantificamos el número de las cláusulas y el número de cada tipo de procesos en los dos córpora. Después dividimos las cláusulas en las que expresan evaluación y aquellas que son neutras; las clausulas con evaluación fueron clasificadas en tres grupos de acuerdo con la división del sistema de actitud en los subtipos: afecto, apreciación y juicio. Al final realizamos un análisis contrastivo, en el cual comparamos los resultados de nuestros dos córpora (preguntas-respuestas y ensayos).

Para codificar la evaluación intentamos usar los mismos criterios que ya se habían empleado a los diferentes córpora en inglés (Hood, 2010). En el proceso de decisión entre diferentes categorías de análisis usamos básicamente criterios conceptuales; utilizamos el test de paráfrasis o sinónimos y acudimos a las autoridades en búsqueda de ejemplos similares en los libros de texto (O’Donnell, Zapavigna \& Whitelaw, 2008). Sería importante mencionar aquí que las dificultades inherentes al análisis valorativo ya fueron reconocidos en la literatura sobre la valoración (Thompson, 2014; Fuoli, 2018). Para reducir la subjetividad del proceso analítico y optimizar su transparencia y confiabilidad, usamos algunas de las recomendaciones de los autores citados, i. e. elaboramos criterios internos aplicables a nuestros córpora, los cuales fueron utilizados por todos los participantes del proyecto. Para los casos difíciles, organizamos discusiones dentro de las reuniones del proyecto donde tratamos de llegar a soluciones y acuerdos, ya que estos acuerdos entre los analistas (inter-coder agreement) llevan a la posibilidad de repetir el análisis de los mismos textos con los 
resultados semejantes (Fuoli, 2018) y, de esta manera, incrementar la objetividad y la confiabilidad del proceso analítico.

\section{Análisis de datos}

\subsection{Análisis de transitividad}

Primero contamos el número de palabras y cláusulas en nuestros córpora, así como registramos la frecuencia de procesos verbales, relacionales y mentales en estas cláusulas. Los resultados se muestran en la Tabla 1.

Tabla 1. Frecuencia de procesos en nuestros córpora.

\begin{tabular}{|c|c|c|c|c|c|c|c|c|c|}
\hline Textos & Palabras & Cláusulas & Procesos & \multicolumn{2}{|c|}{ RELACIONAL } & \multicolumn{2}{|c|}{ VERBL } & \multicolumn{2}{c|}{ MENTAL } \\
\hline & $\mathrm{N}$ & $\mathrm{N}$ & $\mathrm{N}$ & $\mathrm{N}$ & $\%$ & $\mathrm{~N}$ & $\%$ & $\mathrm{~N}$ & $\%$ \\
\hline P-R (10) & 5071 & 547 & 261 & 114 & $44 \%$ & 93 & $36 \%$ & 54 & $21 \%$ \\
\hline En (10) & 18883 & 2297 & 983 & 480 & 48.8 & 280 & 28.5 & 223 & 22.7 \\
\hline
\end{tabular}

Se puede apreciar en la Tabla 1 que los procesos analizados en el corpus PreguntaRespuesta (P-R) equivalen a 261 y a 983 en el corpus Ensayo (En). Se ve claramente que aunque el número de textos es igual en cada corpus, los del corpus En son bastante más extensos: 5071 palabras en el corpus P-R vs. 18883 palabras en el corpus En, es decir, el segundo corpus es más que tres veces más grande que el primero, lo cual se puede explicar por sus características genéricas. En cuanto a los tipos de procesos, en el corpus P-R 114 de estos son relacionales, 93 son verbales y 54, mentales, mientras que en el corpus En 480 son relacionales, 280, verbales y 223, mentales.

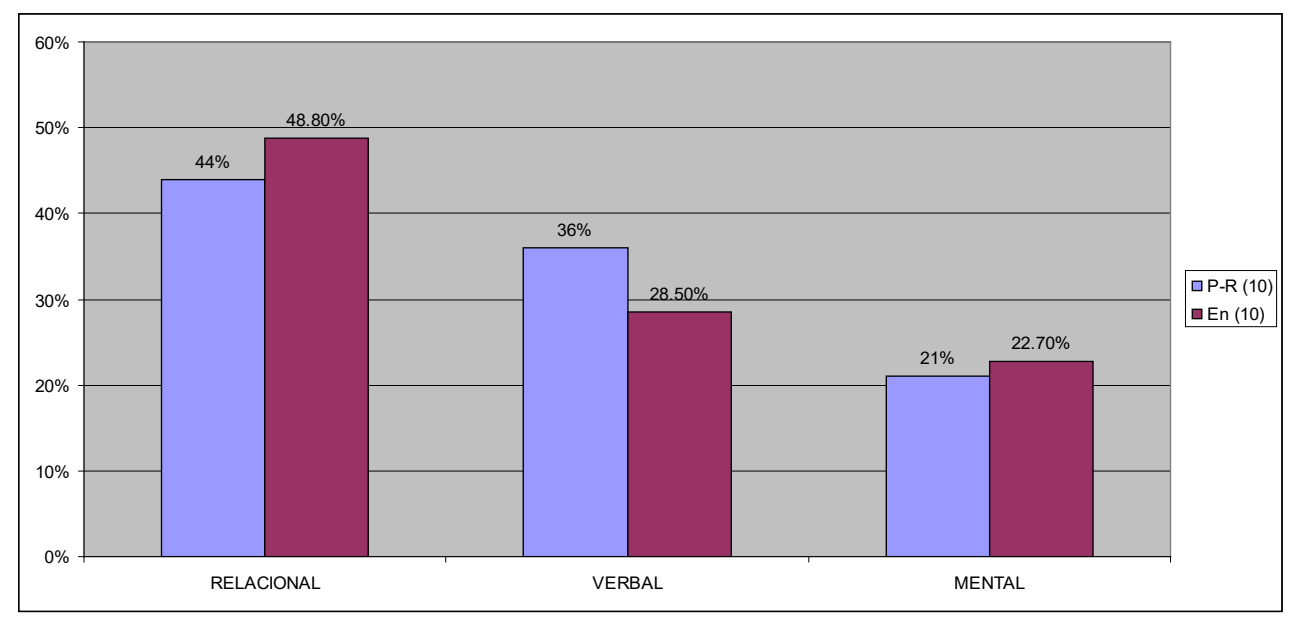

Gráfico 1. Frecuencia de cada tipo de proceso en nuestros córpora.

Ea Gráfico 1 presenta la frecuencia total de cada tipo de proceso, se observa que los procesos relacionales predominan en los dos córpora aun cuando su porcentaje es más alto en el corpus En (48,8\%) en comparación con los textos P-R (44\%). Los 
procesos verbales están en el segundo lugar, en este caso su porcentaje crece más en el grupo P-R $(36 \%)$ y disminuye en el grupo En $(28,5 \%)$. Los procesos mentales son menos frecuentes en los dos córpora y ocupan el tercer lugar, además su porcentaje es similar en ambos grupos de textos ( $21 \%$ vs. $22,7 \%)$.

\subsection{Análisis de actitud}

El siguiente paso fue analizar todas las cláusulas desde el punto de vista de actitud y dividirlas en dos grupos: aquellas que expresan significados evaluativos y las que no lo hacen. La Tabla 2 nos provee los resultados de este análisis.

Tabla 2. Frecuencia de cláusulas con y sin evaluación.

\begin{tabular}{|c|c|c|c|c|c|}
\hline Textos & Procesos & \multicolumn{2}{|c|}{ Procesos CON evaluación } & \multicolumn{2}{c|}{ Procesos SIN evaluación } \\
\hline & & $\mathrm{N}$ & $\%$ & $\mathrm{~N}$ & $\%$ \\
\hline P-R & 261 & 65 & $\mathbf{2 5} \%$ & 196 & $\mathbf{7 5} \%$ \\
\hline En & 983 & 336 & $\mathbf{3 4} \%$ & 647 & $\mathbf{6 6} \%$ \\
\hline
\end{tabular}

Como se deduce de la Tabla 2, en los dos grupos de textos prevalecen las cláusulas neutras, es decir, sin ningún tipo de evaluación. Así, en el corpus P-R el número de las cláusulas sin evaluación triplica el de las cláusulas evaluativas, en otras palabras, tan solo un cuarto del número total de cláusulas (25\%) contienen elementos evaluativos. En el corpus En el número de cláusulas valorativas aumenta en comparación con el corpus P-R, pero aun así las cláusulas neutras duplican el número de las cláusulas con evaluación, estas últimas constituyen aproximadamente una tercera parte $(34 \%)$ del número total de cláusulas.

Después, dividimos las cláusulas que contienen evaluación en dos grupos de acuerdo con su orientación o polaridad: positivas y negativas, los resultados son disponibles en el Gráfico 2.

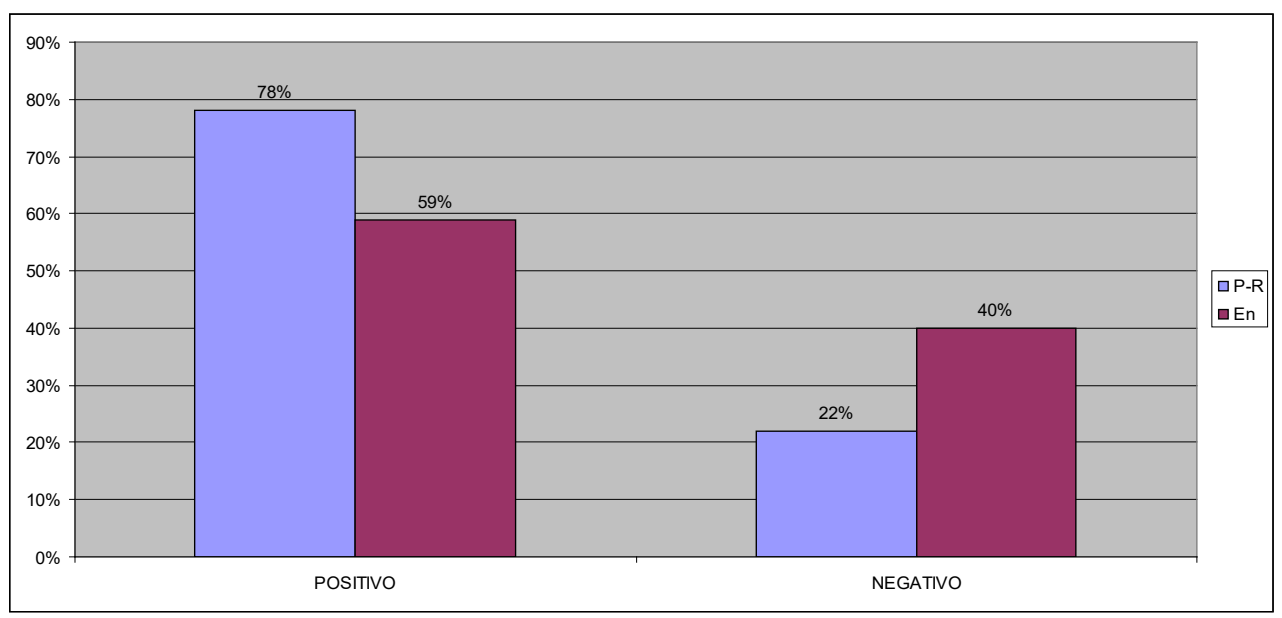

Gráfico 2. Porcentaje de evaluación positiva y negativa en los dos córpora. 
Como se muestra en el Gráfico 2, la evaluación positiva domina en ambos córpora: con 78\% en el corpus P-R y 59\% en el corpus En, se puede ver que el porcentaje de la evaluación negativa es más alto en los textos del género Ensayo, lo cual podría significar que los estudiantes se ponen más críticos cuando escriben ensayos.

A continuación, identificamos y contamos todos los casos de los tres dominios de actitud (afecto, apreciación, juicio) en los dos córpora para ver qué recursos valorativos prefieren los estudiantes, los resultados se puede apreciar en la Tabla 3.

Tabla 3. Los tres dominios de actitud en los dos córpora.

\begin{tabular}{|c|c|c|c|c|}
\hline & afecto & apreciación & juicio & \\
\hline P-R & $4,6 \%$ & $80 \%$ & $15,4 \%$ & $100 \%$ \\
\hline En & $2,7 \%$ & $71,4 \%$ & $25,9 \%$ & $100 \%$ \\
\hline
\end{tabular}

Llama la atención la preponderante posición de apreciación entre otros dominios de actitud en ambos grupos de textos. En los textos pregunta-respuesta llega a 80\% de todas las cláusulas valorativas, en los ensayos su número es un poco menos, pero también muy alto (71,4\%). El juicio que ocupa el segundo lugar tiene los números mucho más modestos (15,4\% para los textos P-R vs. 25,9\% para los textos En). En cuanto al afecto, este resulta ser el recurso menos escogido por los estudiantes (con $4,6 \%$ en el corpus P-R y 2,7\% en el corpus En), se puede concluir que los escritores recurren muy rara vez a él.

\subsection{Correlación de los sistemas de transitividad y actitud}

En la siguente etapa de análisis tratamos de detectar cuál o cuáles tipos de procesos se asocian más con la evaluación (Tabla 4). Se puede observar en la Tabla 4 que las cláusulas relacionales con actitud constituyen 11\% del número total de cláusulas analizadas del corpus P-R, las verbales con actitud, 8,4\%, y las mentales con actitud, $5,4 \%$, mientras que en el corpus En los números respectivos para los tres tipos de cláusulas son $20 \%$ vs. $6 \%$ vs. $7,4 \%$. De esta manera, en el grupo de textos preguntarespuesta el primer lugar lo llevan las cláusulas relacionales, en el segundo están las verbales y en el tercero, las mentales. Esta secuencia cambia en el grupo de ensayos: el primer lugar, al igual que en el otro grupo, lo tienen las cláusulas relacionales, pero el segundo lugar, lo ocupan aquí las cláusulas mentales y en el tercero están las verbales. Además es notorio el crecimiento de las cláusulas relacionales con actitud en el corpus de ensayos $(20 \%)$ en comparación con el corpus de preguntas-respuestas $(11 \%)$.

Tabla 4. Frecuencia de cláusulas con y sin evaluación dentro de cada corpus.

\begin{tabular}{|c|c|c|c|c|c|c|}
\hline Textos & $\begin{array}{c}\text { Relacionales } \\
\text { con actitud }\end{array}$ & $\begin{array}{c}\text { Relacionales } \\
\text { sin actitud }\end{array}$ & $\begin{array}{c}\text { Verbales } \\
\text { con actitud }\end{array}$ & $\begin{array}{c}\text { Verbales } \\
\text { sin actitud }\end{array}$ & $\begin{array}{c}\text { Mentales } \\
\text { con actitud }\end{array}$ & $\begin{array}{c}\text { Mentales } \\
\text { sin actitud }\end{array}$ \\
\hline P-R & $29(11 \%)$ & $85(32,5 \%)$ & $22(8,4 \%)$ & $71(27 \%)$ & $14(5,4 \%)$ & $40(15 \%)$ \\
\hline En & $198(20 \%)$ & $282(29 \%)$ & $61(6 \%)$ & $215(22 \%)$ & $73(7,4 \%)$ & $150(15 \%)$ \\
\hline
\end{tabular}


La relación entre la actitud y el tipo de cláusula descrita arriba se presenta de la manera visual en el Gráfico 3 donde se observa, en primer lugar, la predominancia de cláusulas no valorativas y, en segundo lugar, los porcentajes de tres tipos de cláusulas que codifican algún tipo de evaluación.

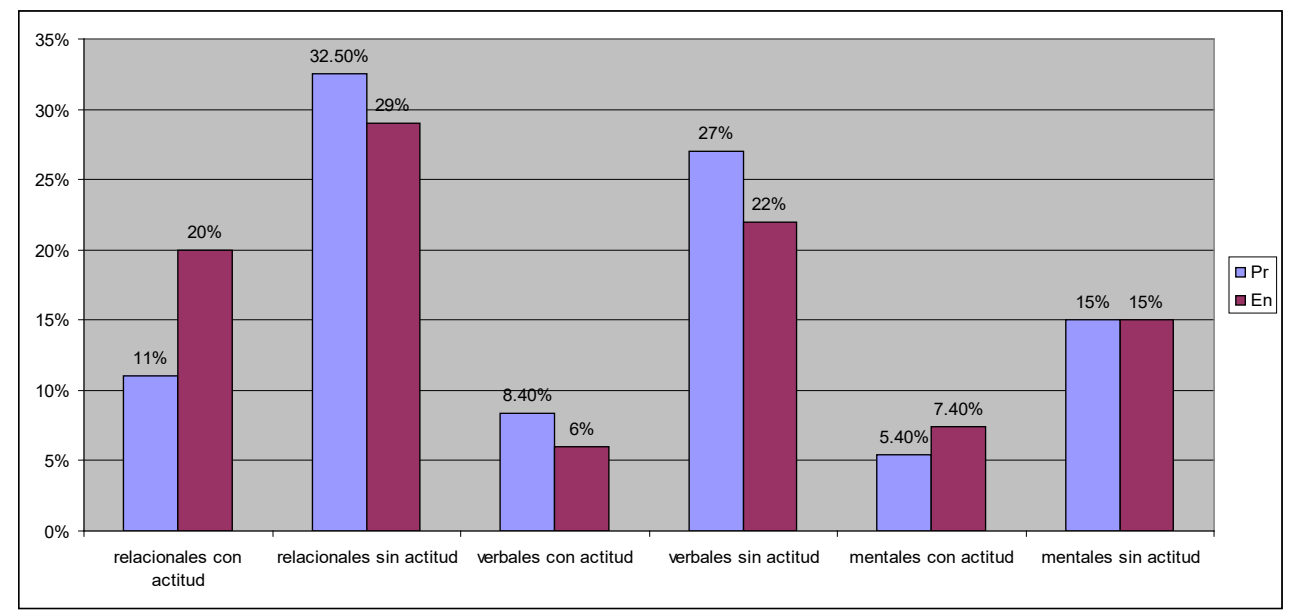

Gráfico 3. Tres tipos de cláusulas en relación a evaluación.

El Gráfico 4 muestra cómo las tres áreas de actitud se distribuyen entre las cláusulas que expresan valoración en ambos córpora. Se puede deducir de el Gráfico 5 que en el corpus P-R la apreciación supera las otras dos áreas (afecto y juicio) en los tres tipos de cláusulas analizadas: entre las cláusulas relacionales 83\% expresan apreciación, $14 \%$ juicio y solo $3 \%$ afecto; en el grupo de procesos verbales tenemos $77 \%$ de apreciación, $23 \%$ de juicio y no hay casos de afecto; finalmente, entre las cláusulas mentales hay $79 \%$ de apreciación, $7 \%$ de juicio y $14 \%$ de afecto.

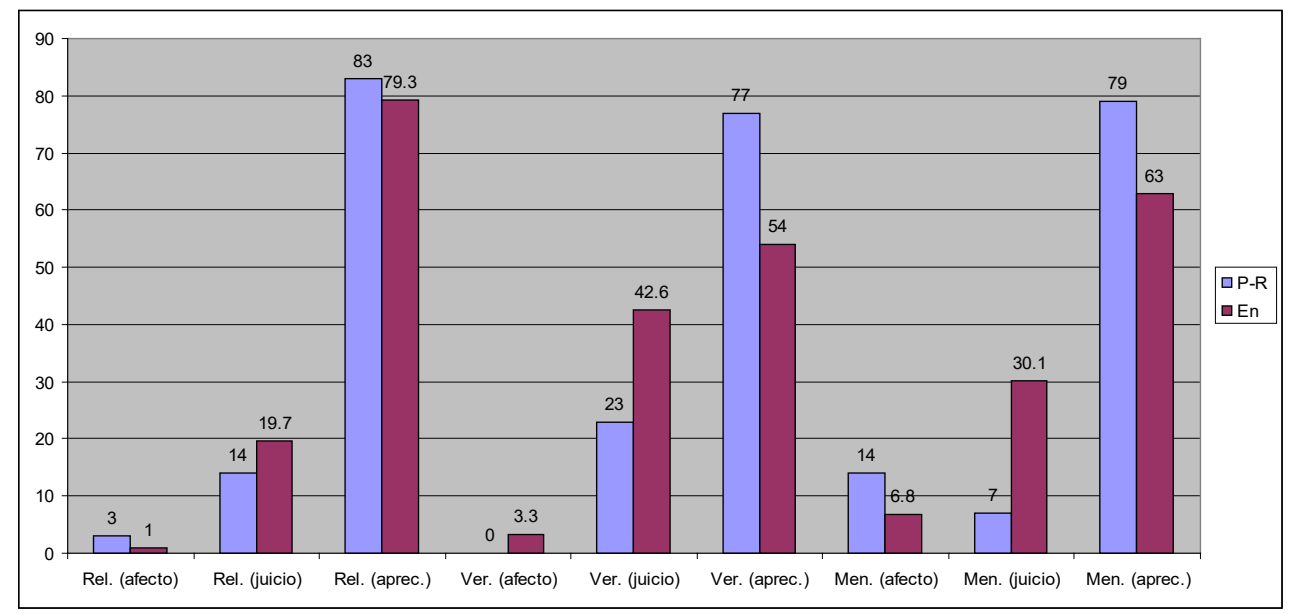

Gráfico 4. Tres áreas de actitud en relación a los tres tipos de procesos. 
En el corpus de ensayos (En) el cuadro también es muy parecido, en los tres tipos de las cláusulas sale la apreciación dominante con mucha ventaja: 79,3\% entre las cláusulas relacionales versus $19,7 \%$ de juicio and $1 \%$ de afecto; $54 \%$ entre las cláusulas verbales versus $42,6 \%$ de juicio y 3,3\% de afecto; $63 \%$ entre las cláusulas mentales versus 30,1\% de juicio y $14 \%$ de afecto. Como consecuencia, la apreciación ocupa el primer lugar aquí, al igual que en el corpus P-R. Vale la pena notar que en ambos córpora prevalecen las cláusulas relacionales con apreciación. Es notorio también que en el corpus En se incrementa considerablemente la incidencia de juicio, sobre todo en cláusulas verbales y mentales.

\subsection{Realización lingüística de ACTITUD en los dos córpora}

Finalmente, registramos la realización de actitud en nuestros córpora en términos de categorías lingüísticas descritas arriba. Los resultados se puede observar en el Gráfico 5. El atributo resulta ser la expresion más típica de actitud en los textos estudiantiles de ambos córpora. Le sigue la cláusula proyectada en el corpus P-R, pero en el corpus En el segundo lugar lo ocupa el adjunto, mientras que en la tercera posición sucede vice versa, es decir, allí se encuentra el adjunto, en el corpus P-R, y la cláusula proyectada, en el corpus En. De esta manera, el atributo, la cláusula proyectada y el adjunto se colocan en los primeros tres lugares en ambos córpora.

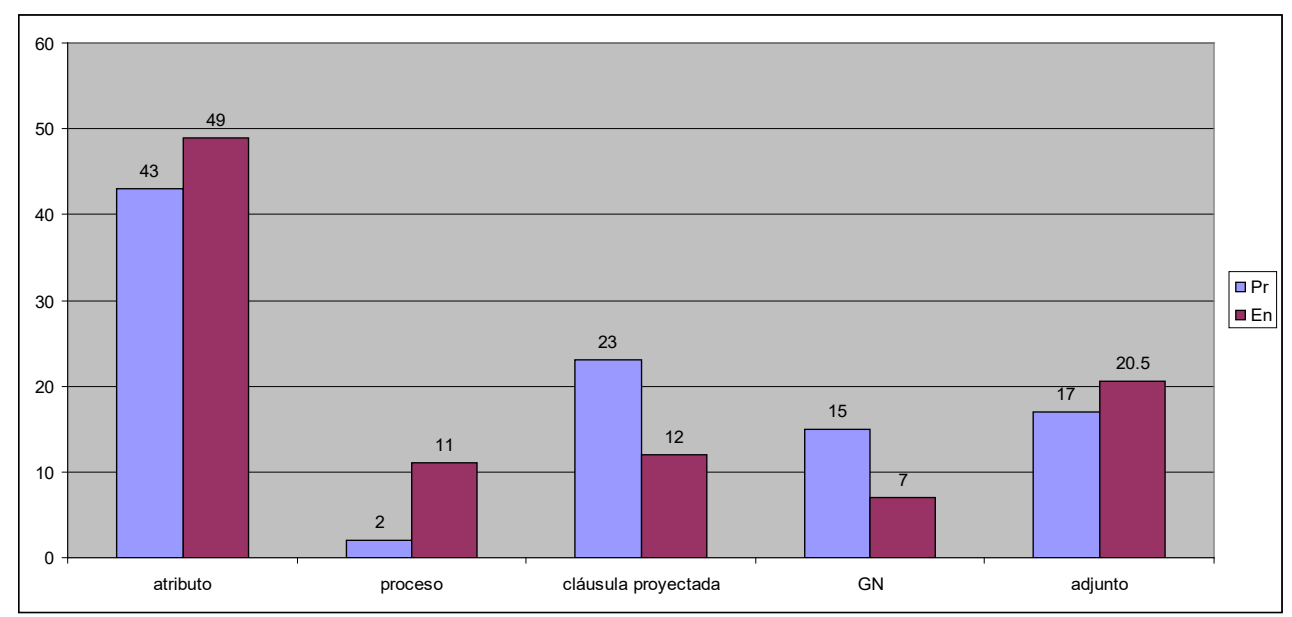

Gráfico 5. Realización lingüística de actitud en nuestros córpora.

En cuanto al proceso, este resulta el recurso menos usado en el corpus P-R, mientras que en el corpus En se acerca al uso de la cláusula proyectada. De la misma manera, el grupo nominal es la realización menos frecuente en el corpus En, pero el corpus P-R sigue de cerca al adjunto. Entonces, las diferencias principales entre los dos corpora conciernen básicamente dos categorías: el proceso y el grupo nominal. 


\section{Discusión}

En este trabajo intentamos examinar la relación entre los tipos de procesos a nivel léxico-gramatical y la expresión de evaluación a nivel semántico-discursivo en la redacción académica de los estudiantes de licenciatura en historia. Tuvimos como objetivo explorar cómo las opciones que hacen estudiantes del sistema de transitividad interactúan con sus opciones del Sistema de Valoración. También queríamos indagar si estas opciones cambian dependiendo del género discursivo del escrito, por lo cual escogimos dos géneros diferentes para nuestro análisis.

El análisis ideacional realizado vía el sistema de transitividad que llevamos a cabo en esta investigación mostró mucha similitud entre los dos grupos de textos: tanto en los textos pregunta-respuesta como en los ensayos los procesos predominantes han sido los relacionales, los verbales están en el segundo lugar y los menos frecuentes resultan los procesos mentales. Al mismo tiempo hay que remarcar que los porcentajes no son iguales, en los ensayos el porcentaje de procesos relacionales es más elevado que en los textos pregunta-respuesta, lo cual puede significar que los primeros se acercan más a la escritura científica, cuya característica importante es justamente la abundancia de procesos relacionales. Por otra parte, el porcentaje de procesos verbales es más alto en los textos pregunta-respuesta en comparación con los ensayos, que se puede interpretar como la expresión de un estilo más informal o más coloquial, según nuestros estudios previos sobre los procesos verbales (Ignatieva, 2016). En cuanto a los procesos mentales, su número es similar en los dos grupos de textos.

Analizando el Sistema de Valoración pudimos observar otras similitudes entre los dos grupos de textos. Así, en ambos córpora predominan cláusulas neutras, es decir, cláusulas que no contienen elementos evaluativos (75\% en el corpus P-R y 66\% en el corpus En), esto significa que solo una cuarta parte de las cláusulas en el corpus P-R y una tercera parte del corpus En expresan actitud. Sin embargo registrando las diferencias, se hace evidente que el número de cláusulas con valoración crece en el grupo de ensayos en comparación con el grupo de preguntas-respuestas, lo cual se puede interpretar como un incremento de la capacidad evaluativa de los alumnos al redactar textos de este género.

Podemos notar también que expresando la valoración los estudiantes en general se inclinan más hacia una evaluación positiva en comparación con la negativa; esta tendencia de evaluar cosas, eventos y personajes de manera positiva es el otro punto donde los dos córpora son similares. Empero, los números se distinguen: mientras que en el corpus P-R solo una cuarta parte de las cláusulas evaluativas son negativas, en el corpus En esta correlación no es tan drástica: tenemos aproximadamente $60 \%$ de cláusulas positivas contra $40 \%$ de las negativas. Estos hallazgos se pueden explicar por 
la suposición de que la capacidad crítica de los estudiantes en general no está plenamente desarrollada, sin embargo entra más en juego cuando se trata de género de ensayos.

En cuanto a los tres dominios de actitud, la apreciación tiene una aplastante mayoría en las cláusulas valorativas de los dos córpora, aun cuando el número de cláusulas con apreciación decrece un poco en el corpus En, lo cual permite que crezca más la cantidad de casos de juicio en el grupo de ensayos.

Cuando consideramos el tipo de proceso que se relacióna más con los aspectos evaluativos, detectamos que los procesos relacionales se ubican en el primer lugar, en ambos córpora, seguidos por los verbales y los mentales que están en tercer lugar. Esto no es sorprendente si tomamos en cuenta la semántica de los procesos relacionales, que expresan un estado de cosas, frecuentemente sujeto a la evaluación, lo cual explica su amplio uso en textos históricos (Coffin, 2006; Moss, 2010). Además los procesos relacionales ayudan en generar el carácter 'objetivo' del discurso histórico (Oteíza Silva, 2006).

La cuestión de tipos de procesos asociados más con la evaluación está muy conectada con las tres áreas del sistema de actitud. Por ejemplo, los procesos relacionales están ligados con apreciación como el recurso favorito para expresar valoración, mientras que el juicio se liga más con procesos verbales. Sería lógico entonces esperar la apreciación en el primer lugar, y como ya lo vimos, así sucede en nuestros córpora: la apreciación sobrepasa considerablemente al juicio que le sigue mientras que el afecto se ubica en el último lugar en los tres tipos de cláusulas para ambos córpora. Se puede deducir que los textos estudiantiles de historia realizan la valoración básicamente a través de apreciación (ligada a procesos relacionales), esto está asociado con que los estudiantes de esta disciplina tienden a evaluar hechos, sucesos y eventos y, significativamente menos, figuras históricas. A pesar de que la secuencia de las tres áreas de actitud es la misma en los dos grupos de textos (apreciación $>$ juicio $>$ afecto, en el orden decreciente) vale la pena subrayar que el porcentaje de cláusulas con juicio crece considerablemente en los ensayos en comparación con los textos pregunta-respuesta, sobre todo en las cláusulas verbales y mentales, lo cual contribuye a las características críticas de la escritura estudiantil.

La exploración de la realización lingüística de la actitud ha revelado que el atributo resulta ser el recurso más frecuente de la expresión de valoración en ambos córpora, lo cual ya se esperaba tomando en cuenta que el atributo de la manera característica se asocia más con procesos relacionales, mismos que han tenido una alta frecuencia en nuestros córpora.

El segundo y tercer lugar lo ocupan la cláusula proyectada y el adjunto aunque en diferente orden de secuencia en los dos grupos de textos. Como ya lo mencionamos en el apartado 3, procesos verbales y mentales poseen una capacidad proyectante y la 
proyección frecuentemente actúa para construir el significado interpersonal (Thompson, 2004) y sirve para codificar la actitud o posicionamiento del escritor (Forey, 2008). Es evidente la relación estrecha entre los procesos verbales o mentales en la cláusula proyectante y la propia cláusula proyectada, siendo esta relación un ejemplo interesante de la compleja interacción de significados ideacionales (transmitidos por el proceso) e interpersonales (transmitidos por la cláusula proyectada), ya que las cláusulas proyectantes funcionan como "un lente interpersonal a través del cual se interpreta la cláusula proyectada" (Coffin \& Hewings, 2004: 162).

\section{CONCLUSIÓN}

Entonces, nuestro análisis puso en evidencia el hecho de que hay muchas similitudes entre los textos pregunta-respuesta y los ensayos en el área de historia: prevalencia de procesos relacionales, de cláusulas sin valoración, de apreciación en comparación con otras áreas de actitud, de atributo como el medio más frecuente para expresar los aspectos evaluativos. Este resultado fue un tanto inesperado ya que pensamos encontrar más diferencias entre los dos géneros académicos. Pero si tomamos en cuenta que los alumnos no habían recibido ninguna indicación genérica antes de elaborar estos escritos, los resultados no deben sorprendernos, los alumnos, probablemente, no estaban conscientes de que se trata de dos tipos de textos distintos con sus características genéricas específicas. De ahí, se hace evidente la necesidad de una instrucción genérica basada en la Pedagogía del Género (Rose \& Martin, 2012) para los alumnos que estudian carreras universitarias y tienen que redactar diferentes tipos de textos (expositivos, explicativos y argumentativos, entre otros) en sus disciplinas ${ }^{3}$.

En cuanto a las diferencias, estas tienen un carácter cuantitativo más bien: en el corpus de ensayos en comparación con el corpus P-R incrementa el número de cláusulas valorativas en general y de negativas, en particular, y crece el papel de juicio, como índices de la capacidad crítica de estudiantes. De esta manera, la pertenencia de escritos a una misma disciplina, en este caso, a la historia, parece tener un papel más decisivo en la selección de recursos lingüísticos en la redacción, en comparación con su pertenencia a un género discursivo. Esto va de acuerdo también con nuestro estudio previo que mostró diferencias significativas en dos grupos de textos pertenecientes a un solo género, pero a dos disciplinas diferentes: literatura e historia (Ignatieva, 2019).

Para concluir, el análisis llevado a cabo en este proyecto nos permitió observar algunos rasgos genéricos de escritos estudiantiles de historia como, por ejemplo, el uso extenso de procesos relacionales ligados a la apreciación como el recurso preferido de valoración, lo cual se podría explicar por el carácter estático y 'objetivo' de los textos estudiantiles y la tendencia de evaluar los hechos y sucesos más que a los personajes 
históricos (véase también Moss \& Chamorro Miranda, 2011). De esta manera, las tendencias que encontramos en la interacción de sistemas de transitividad y valoración $\mathrm{y}$, consecuentemente, de los significados ideacionales e interpersonales, asimismo que de los aspectos léxico-gramaticales y semántico-discursivos de textos académicos muestran conclusiones interesantes, pero necesitan confirmarse explorando más córpora y comparando datos de diferentes géneros y disciplinas. Estos hallazgos también tienen importantes implicaciones pedagógicas y pueden encontrar su aplicación práctica en la enseñanza de escritura académica a través de disciplinas universitarias.

\section{REFERENCIAS BIBLIOGRÁFICAS}

Caffarel, A. (2006). A systemic functional grammar of French: From grammar to discourse. Londres: Continuum.

Castro Azuara, C. (2013). Posicionamiento discursivo en el ensayo de opinión escrito por estudiantes universitarios. Lenguas en Contexto, 10, 98-107.

Chen, L. (2007). Analysing attitude: Positive verbal process sub-functions and media bias. RASK, International Journal of Language and Communication, 25, 25-55.

Coffin, C. (2006). Historical discourse. The language of time, cause and evaluation. Londres: Continuum.

Coffin, C. \& Hewings, A. (2004). IELTS preparation for tertiary writing: Distinctive interpersonal and textual strategies. En L. Ravelli \& R. Ellis (Eds.), Academic writing in context: Social-functional perspectives on theory and practice (pp. 153-171). Londres: Continuum.

Forey, G. (2008). Projecting clauses: Interpersonal realization of control and power in workplace texts. En G. Forey \& G. Thompson (Eds.), Text type and texture (pp. 151-172). Londres: Equinox.

Fuoli, M. (2018). A step-wise method for annotating APPRAISAL. Functions of Language, 25(2), 1-27.

García, A. (2013). Construing experience in Spanish: Revisiting a systemic functional description of Spanish nuclear transitivity. Revista Signos. Estudios de Lingüistica, 46(81), 29-55.

Ghio, E. \& Fernández, M. D. (2008). Manual de Lingüistica Sistémico-Funcional: El enfoque de M. A. K. Halliday y R. Hasan: Aplicaciones a la lengua española. Santa Fe, Argentina: Universidad Nacional del Litoral.

Halliday, M. A. K. (1968). Notes on transitivity and theme in English. Journal of Linguistics, 4, 179-215. 
Halliday, M. A. K. (1976). System and function in language. Londres: Oxford University Press.

Halliday, M. A. K. (1978). Language as social semiotic: The social interpretation of language and meaning. Londres: Edward Arnold.

Halliday, M. A. K. (1994). An Introduction to Functional Grammar. Londres: Edward Arnold.

Halliday, M. A. K. \& Matthiessen, C. M. I. M. (1999). Construing experience through meaning: A language-based approach to cognition. Londres: Continuum.

Halliday, M. A. K. \& Matthiessen, C. M. I. M. (2004). An Introduction to Functional Grammar. 3er Edition. Londres: Edward Arnold.

Hood, S. (2010). Appraising research: Evaluation in academic writing. Londres: Palgrave Macmillan.

Hunston, S. (1995). A corpus study of some English verbs of attribution. Functions of Language, 2(2), 133-158

Hyland, K. (1999). Academic attribution: Citation and the construction of disciplinary knowledge. Applied Linguistics, 20(3), 341-367.

Ignatieva, N. (2011). Verbal processes in student academic writing in Spanish from a systemic functional perspective. Lenguaje, 39, 447-467.

Ignatieva, N. (2016). Reflexiones sobre los procesos verbales en el marco sistémico. En N. Ignatieva \& D. Rodríguez Vergara (Eds.), Lingüistica Sistémico-Funcional en México: Aplicaciones e implicaciones (pp. 35-48). México: Universidad Nacional Autónoma de México.

Ignatieva, N. (2019). Transitive and attitudinal aspects in a functional analysis of academic discourse in Spanish. Open Journal of Modern Linguistics, 9, 165-178.

Ignatieva, N. \& Zamudio, V. (2012). Perspectiva funcional de los procesos verbales en los escritos estudiantiles de literatura e historia en español. DELTA, 28, 561579 .

Ignatieva, N. \& Colombi, M. C. (Eds.) (2014). CLAE: Corpus del Lenguaje Académico en Español de México y los Estados Unidos: Un análisis sistémico funcional. México: Universidad Nacional Autónoma de México.

Ignatieva, N. \& Rodríguez-Vergara, D. (2015). Verbal processes in academic language in Spanish: Exploring discourse genres within the systemic functional framework. Functional Linguistics, 2(2), 1-10. 
Lavid, J. (2008). The grammar of emotion in English and Spanish: A systemicfunctional approach. En C. Jones \& E. Ventola (Eds.), From language to multimodality: New developments in the study of ideational meaning (pp. 67-85). Londres: Equinox.

Lavid, J, Arús, J. \& Zamorano, J. R. (2010). Systemic functional grammar of Spanish: A contrastive study with English. Londres \& Nueva York: Continuum.

Martin, J. R. (1992). English text. Filadelfia: John Benjamins.

Martin, J. \& Rose, D. (2003). Working with discourse: Meaning beyond the clause. Londres \& Nueva York: Continuum.

Martin, J. R. \& White, P. R. (2005). The language of evaluation. Nueva York: Palgrave Macmillan.

Matthiessen, C. M. I. M. (1995). Lexicogrammatical cartography: English systems. Tokyo: International Language Sciences.

Matthiessen, C. M. I. M. (1999). The system of transitivity: An exploratory study of text-based profiles. Functions of Language, 6(1), 1-51.

Matthiessen, C. M. I. M., Teruya, K. \& Lam, M. (2010). Key terms in systemic functional linguistics. Londres: Continuum.

Montemayor-Borsinger, A. (2009). Tema: Una perspectiva funcional de la organización del discurso. Universidad de Buenos Aires: Eudeba.

Moss, G. (2010). Textbook language, ideology and citizenship. The case of a history book in Colombia. Functions of Language, 17(1), 71-93.

Moss, G. \& Mizuno, J. (2011). Las voces del texto. En N. Barletta Manjarrés \& D. Chamorro Miranda (Eds.), El texto escolar y el aprendizaje: Enredos y desenredos (pp. 149-179). Barranquilla, Colombia: Editorial Universidad del Norte.

Moss, G. \& Chamorro Miranda, D. (2011). La pedagogía de la simplificación: La enseñanza de historia por medio de pista y pezca. En (Re)Construcción: Discurso, identidad y nación en los manuales escolares de bistoria y de ciencias sociales (pp. 269320). Chile: Editorial Cuarto Propio.

Moyano, E. I. (2013). El lenguaje de las disciplinas y los géneros de su recontextualización escolar. Una aproximación desde la lingüística sistémicofuncional. En E. Moyano (Coord.), Aprender ciencias y humanidades: Una cuestión de lectura y escritura: Aportes para la construcción de un programa de inclusión social a través de la educación lingüística (pp. 31-78). Los Polvorines: Universidad Nacional de General Sarmiento. 
Navarro, F. (2014). Gradación y compromiso en escritura académica estudiantil de humanidades. Análisis contrastivo desde la Teoría de la Valoración. Estudios de Lingüística Aplicada, 60, 9-33.

O’Donnell, M., Zappavigna, M. \& Whitelaw, C. (2008). A survey of process type classification over difficult cases. En C. Jones \& E. Ventola (Eds.), From Language to Multimodality: New Developments in the Study of Ideational Meaning (pp. 47-64). Londres: Equinox.

Oteíza Silva, T. (2006). El discurso pedagógico de la historia: Un análisis lingüístico sobre la construcción ideológica de la historia de Chile (1970-2001). Santiago de Chile: Frasis.

Perales-Escudero, M. D. (2018). Writer-reader interaction in economics abstracts in English and Spanish; Implications for teaching and translation. MEXTESOL Journal, 42(2), 1-16.

Rose, D. \& Martin, J. R. (2012). Learning to write, reading to learn: genre, knowledge and pedagogy in the Sydney school. Londres: Equinox.

Rodríguez-Vergara, D. \& Contijoch Escontria, M. C. (2016). Transitividad y valoración de la actitud en ensayos de geografía: Un análisis sistémicofuncional. Signos Lingüisticos, XII(23), 8-29.

Thompson, G. (1996). Introducing Functional Grammar. Londres: Edward Arnold.

Thompson, G. \& Hunston, S. (2000). Evaluation: An introduction. En S. Hunston \& G. Thompson (Eds.), Evaluation in text: Authorial stance and the construction of discourse (pp. 1-27). Oxford: Oxford University Press.

Thompson, G. (2014). Affect and emotion, target-value mismatches, and Russian dolls: Refining the appraisal model. En G. Thompson \& L. Alba-Juez (Eds.), Evaluation in Context (pp. 47-66). Ámsterdam: John Benjamins.

Zamudio, V. (2016). La expresión de opiniones y puntos de vista en textos académicos estudiantiles sobre literatura. Lenguaje, 44(1), 35-39.

\section{NOTAS}

1 Esta investigación se está llevando a cabo gracias al Programa de Apoyo a Proyectos de Investigación e Innovación Tecnológica de la UNAM, en el cual está registrado nuestro proyecto titulado "Tipología verbal y evaluación en la escritura académica de las humanidades: un estudio sistémico-funcional”, con clave de registro IN401716.

${ }^{2}$ No se planea profundizar más sobre el tema de género en este trabajo porque este término al igual que los nombres de los géneros particulares (ensayo, pregunta-respuesta, etc.) se utilizan 
más bien como términos operativos. Partiendo de los datos, aplicamos las etiquetas que se usan en el ambiente universitario para referirse a los textos estudiados.

${ }^{3}$ Cabe mencionar que en el Plan de Estudios del Colegio de Historia no hay ninguna materia que haga una referencia explícita a la escritura académica en la historia, mientras que dentro de la lingüística educativa de corte sistémico-funcional se ha insistido en la importancia de la ‘enseñanza explícita del discurso académico' (Rose \& Martin, 2012; Moyano, 2013). Partiendo de estas necesidades, la escuela donde trabajamos (Escuela Nacional de Lenguas, Lingüística y Traducción de la UNAM) tiene planes de crear un centro de escritura académica para estudiantes universitarios con el objetivo de reforzar las habilidades de escritura en la alfabetización académica avanzada. 